VII.

\title{
Ueber zwei Fälle von Dementia paralytica mit Hirnsyphilis.
}

\author{
(Pseudoparalysis syphilitica nach Jolly.) \\ Von \\ Dr. Rentsch, \\ Anstaltsarzt der Heil- und Pflegeanstalt Sonnenstein.
}

(Hierzu Tafel IV.)

\begin{abstract}
Den Namen Pseudoparalysis syphilitica will Jolly nur für Fälle angewandt wissen, die "durchaus im ganzen Verlaufe das Bild der Dementia paralytica, sowobl in der agitirten, als in der einfach dementen Form zeigen, bei welchen wir aus den Symptomen niemals das Recht hätten, von Gehirnsyphilis zu sprechen, und bei denen die Sectiou zeigt dass neben den diffusen, degenerativen Processen der feineren Rindenelemente (wie sie der Paralyse zukommen), welche zur Demenz geführt haben, noch locale, zweifellos syphilitische Veränderungen im Gehirn vorhanden sind", Fälle, bei denen man ausser durch den Verlauf und den anatomischen Befund noch durch das therapeutische Experiment zu der Ueberzeugung kommt, dass hier die Syphilis eine directere und unmittelbarere Rolle gespielt haben muss.

Noch ist die Zahl derartiger in der Literatur vorhandenen Fälle eine ziemlich beschränkte; ich kann sie nun durch zwei in der Heilund Pfleganstalt Sonnenstein beobachtete Fälle ergänzen:

1. Fall: Handarbeiter Andreas L., 42 Jahre, wurde der Landesanstalt Sonnenstein am 14. Februar 1901 zugeführt. Nach dem beigebrachten ärztlichen Gutachten erblich nicht belastet, normal intellektuell entwickelt, früher lebhaft, hatte einen geordneten Lebenswandel geführt, war fleissig; trank aber regelmässig viel Bier. Ueber durchgemachte Lues, Kopfverletzungen nichts zu erfahren: Zeigt Symptome progressiver allgemeiner Paralyse: Deutliche Sprachstörung (Silbenstolpern und Paraphasio), Beben der Lippen und Zunge, die stossweise hervorgebracht wird, linke Pupille etwas weiter als rechte, rechte reagirt prompt, linke träge. Ohne dass Patient früher psychisch krank war,
\end{abstract}


wurde am 5. October 1900 von der Umgebung des Kranken ein Schwindelanfall beobachtet, er verlor das Gefühl in der rechten Hand, konnte nur noch ganz unverständlich sprechen, schlief daraufhin viel. Nach 3-4 Tagen wurde bis auf eine leichte Sprachbehinderung nichts Abnormes mehr bemerkt. Am 25. December 1900 redete er plötzlich verwirrt, konnte seiner Arbeit seitdem nicht mehr nachgehen, war stumpf und auffallend ruhig; äusserte, fremde Leute seien im Zimmer, die die Wände anspritzten. Am 3. Januar 1901 in das Stadt-Irren- und Siechenhaus zu Dresden in sebr geistesschwachem, durchaus verwirrtem Zustande gebracht, schlief er viel am Tage, verfiel gegen Abend. ziemlich regelmässig in heftige Aufregungszustände, in welchen er nicht irn Bett zu erhalten war, schrie, tobte unō gewaltthätig wurde. Die Aufregungszustände Jiessen dann an Häufigkeit und Intensität nach. Nach der Ueberführung nach Sonnenstein wurde hier folgender Status aufgenommen:

Gang in mässigem Grade unsicher, geschiebt mit gespreizten Beinen. An der Vorderfläche der Unterschenkel bis markstückgrosse, grauröthlich pigmentirte alte Narben, kleine weissliche an der radialen Seite des rechten Vorderarms, schliesslich mehrere Striae auf der Haut beider Leistengegenden. Am Schädel keine Empfindlichkeit bei Erschütternng und Beklopfen. Rechte Nasolabialfalte gegen links eine Spur verstrichen. Zunge weicht eine Spur nach links $a b$, zeigt fibrilläre Zuckungen. Geschmaoksempfindung für sauer nnd bitter normal, für süss und salzig abgeschwächt. Keine Schlingbeschwerden.

In den Nasensepten keine Defecte, Geruchsempindung normal.

Geringer Grad von Nystagmus. Pupillen eng, linke etwas weiter als rechte; auf Lichteinfall und Convergenz beiderseits prompte Reaction.

Sehschärfe normal, Gesichtsfeld nicht eingeschränkt. Fartıensinn normal.

Genitalien 0. B., insbesondere keine Narbe an Glans oder Praeputium nachweisbar. Keine Blasen- und Darmstörungen.

Tastempfindnng normal. Schmerzempfindung an Rumpf und Extremitäten herabgesetzt; auch localisirt Patient am Rumpfe, sowie an Ober- und Untersohenkel Berührungen z. Th. bis auf $10 \mathrm{~cm}$ Entfernung fehlerhaft.

Austrittsstellen des Nerv. V stark druckempfindlich. Romberg in geringem Grade ausgeprägt.

Bauchdeckenreflexe abgeschwächt, Cremasterreflex fehlt, Fusssohlenreflex. gesteigert, Conjunctivalreflex abgeschwächt.

Grobe Kraft nicht berabgesetzt, Ungeschicklichkeit bei auf Befehl ausgeführten Bewegungen, zunehmend bei geschlossenen Augen.

Ziemlich starker Tremor manuum, mässiger Intentionstremor. Patellarsehnenreflex beträchtlich gesteigert, Fussclonus nicht auslösbar.

Status psychicus: Zunächst bei Bettruhe wechselndes Verhalten, zeitweise ruhig daliegend, ohne der Umgebung viel Aufmerksamkeit zo schenken, stand Patient dann plötzlich ohne Ursache auf, packte seine Bettstücke zusammen, wanderte damit durch den Wachsaal. Dann zeitweise sehr laut und störend.

Sprachstörung motorisch nnd sensorisch. Fehlen von Worten, 
Wiederholen desselben Wortes mehrere Male hintereinander, bis das fehlende Wort gefunden ist, wobei häufig eine deutliche Paraphasie zu Tage tritt. Schon beim Nachsprechen einfacher Worte Silbenstolpern.

Giebt den. Geburtstag richtig, das Alter falsch an, ist zeitlich ganz desorientirt (24. Januar 1891 statt 14. Februar 1901), ohne Krankheitseinsicht, Gedächtniss für Ereignisse der letzten Zeit abgeschwächt. Stand der Kenntnisse dürftig: A BC lückenhaft, Monatsnamen nur in falscher Reihenfolge. Hauptstadt von Preussen ist Breslau! Rechnet $4 \times 6=64,9 \times 11=101$, $14+26=24,13-5=20$ u. s. w.

Stimmung meist gleichmässig apathisch.

13. März 1901. Sprach- und Schreibstörung unter Einfluss von $20 \mathrm{~g}$ Jodkali nach und nach gebessert, Patient klarer, aber noch sehr dement.

14. Juni. In den letzten Tagen steigende Unruhe, wenig beeinflussbar durch Hypnotica, zeitweise Absonderung nöthig. Starke Sprachstörung and Demenz.

11. Juli. Rubiger, apathisch.

31. October. Kleiner Decubitus.

10. November. Fiel bei einem Ohnmachtsanfall hin, erholte sich bald wieder, Decubitus geheilt 1 . December.

17. Februar 1902. Nach kurzem paralytischen Anfall vorübergehende Läbmung und Hypalgesie des rechten Armes. Am nächsten Tage schon ist der Arm wieder gebrauçhsfähig.

1. März. Epileptiformer Anfall obne zurückbleibende Lähmung, völlig interesseloses Verhalten.

10. April. Zerreisst das Hemd, stösst klagende, wimmernde Töne aus, ist völlig dement.

12. Juli. Ruhig völlig theilnahmslos in Bett liegend, spricht nie ein Wort, muss die Nahrung gereicht erhalten. Zerbeisst zwangsmässig das Bettzeug.

20. September. Geht körperlich allmälig mehr und mehr zurück, ab und zu unreinlich, mit Koth schmierend.

15. November. Siecht geistig und körperlich immer mehr und mehr dahin, ist meist sehr unrein, reagirt auf keine Anrede mehr, knirscht unausgesetzt mit den Zähnen, sucht zwangsmässig seine Bettdecke zu zerbeissen, vermag sich in keiner Weise mehr selbstthätig zu bewegen, hütet andauernd das Bett.

31. December. Wurde vor 3 Tagen ganz benommen, blieb ruhig theilnahmlos im Bett liegen, nahm keine Nahrung mehr auf. Es stellte sich Fieber ein $39,5^{\circ}$, Puls wurde schwächer, zunehmender Kräfteverfall. Hịnten unten über beiden Lungen Rasselgeräusche, hier und da auch Schallverkürzung.

31. December Nachmittags erfolgt der Exitus unter den Erscheinungen der Herzschwäche und (einer noch im Fortschreiten begriffenen) Aspirationspneumonie.

Aus dem Bericht der am 2. Januar 1903 rorgenommenen Section möchte jeh das Folgende nur hervorheben: 
Beginnende Fäulniss. Lungen im Zustande gangränescirender Pneumonie. Herz schlaff. Kranzarterien zart, elastisch; ihre Intima grau durchscheinend. und glatt.

Aufsteigende Aorta vollkommen frei von arteriosklerotischen Veränderungen.

Genitalien bieten nichts Besonderes dar.

Innenwand der Brust- und Bauchaorta glatt, grauweiss durchscheinend.

Schädelkappe leicht von der Dura zu trennen, schwer, sehr dick. Dura mater schlaff, innen glatt, sehnig glänzend, grauweiss. Pia mater stark milchig und sulzig getrübt und stark verdickt.

Die an der Basis des Gehirns in den Maschen der Pia enthaltene Flüssigkeit ist klar wie an der Convexität des Gehirns.

Die Arterien an der Basis sind im Allgemeinen zart, elastisch und leer, nur an der Arteria basilaris und deren Aesten bemerkt man mehrere grauweisse mehr oder weniger knotige Auftreibungen und Verdickungen, die sich weich gummiartig anfühlen.

Pia am Stirnhirn stellenweise mit der Rinde verwachsen.

Gehirnwindungen schmal, spitz. Consistenz weich.

Seitenventrikel beträchtlich erweitert, enthalten reichlich seröse Flüssigkeit. Ependym verdickt, blass, stark höckerig.

Im 3. und 4. Ventrikel etwas seröse Flüssigkeit; die Auskleidung ist namentlich im 4. Ventrikel sehr reichlich mit kleinen hellen, derb anzufühlenden Knötchen besetzt.

Auf Durchschnitten: Gewebe des Grosshirns, Hemisphären, Streifen- und Sehhügel überall stark durchfeuchtet, mässig blatreich, ziemlich weich.

Rindensubstanz in derStirngegend sehr beträchtlich verschmälert, stellenweise höchstens $2-3 \mathrm{~mm}$ dick.

Die Sectionsdiagnose lautete: Paralysis progressiva. Gummata arteriae basilaris. Gangränescirende Bronchopneumonie.

Hirnstamm und Kleinhirn werden in 10 proc. Formalinlösung conservirt. Nach einigen Tagen werden die Gefässe an der Basis noch etwas frei präparirt, wobei sich Folgendes zeigt (cf. Fig. 1):

An der Gefässwand der rechten Arteria vertebralis findet sich von der Vereinigungsstelle der beiden vertebrales bis $1 \mathrm{~cm}$ spinalwärts reichend eine Auftreibung von blassgelber Farbe. Dieselbe setzt sich nach unten ziemlich scharf gegen die normale Gefässwand (die blaugrau erscheint) ab. Cerebralwärts setzt sich die Verdickung fort auf die Arteria basilaris in einer Ausdehnung von $17 \mathrm{~mm}$. Auch nach oben setzt sich die Verdickung scharf gegen die normale collabirte Gefässwand ab. Die Verdickung besteht aus zahlreichen, gries- bis hirsekorngrossen Knötchen von elastischer Consistenz, graugelblicher Warbe und leicht durchscheinender Beschaffenheit. Die grösste Dicke hat die Gefässwand der Arteria vertebralis dextra kurz vor dem Uebergang in die Basilaris.

Ein vereinzeltes grieskorngrosses, gelbes Knötchen findet sich noch an 
der rechten Arteria cerebelli superior, $7 \mathrm{~mm}$ rom Abgang aus der Basilaris, ein gleiches an der arteria cerebralis der linken Seite.

Bei Anlegung von Querschnitten durch die erkrankten Gefässe zeigte sich das Lumen der betreffenden Gefässe allenthalben bedeutend verengt, am stärksten an der rechten Arteria vertebralis nahe dem Uebergang in die Basilaris selbst. Hier war das eigentliche Gefässlumen bis auf eine kaum noch stecknadelkopfgrosse durch rothes Blut ausgefüllte Oeffnung reducirt.

Die mikroskopische Untersuohung der betreffenden Gefässe ergab folgende Resultate: Aus einer grösseren Anzâhl von Präparaten brauchen wir nur je eins aus jeder der 4 wichtigsten Schnittebenen beschreiben:

1. Die rechte Árteria vertebralis zeigt an der Stelle, wo die stärkste $O b$ literation sich fand, eine Umwandlung der Intima in eine breite Granulationsmembran, in der Hauptsache bestehend aus concentrischen Ringen spindelförmiger Zellen. Stellenweise bat das Gewebe einen mehr homogenen Charakter, die Zellkerne sind hier nur schwach gefärbt. Innerhalb des neugebildeten Gewebes treten theils runde, theils mehr längliche unregelmässig ausgebuchtete Bluträume hervor, die zum Theil umgrenzt sind von einer einfachen Lage spindelförmiger Zellen; stellenweise ist es zu cavernomartigen Bildungen gekommen. Ausserdem finden sich aber zwischen den hyalin entarteten Partien sowohl einzelne, wie in grösserer Menge angehäufte Blutkörperchen. Um einzelne dieser neugebildeten Bluträume herum findet sich eine dichte Anhäufung kleiner Rundzellen; einzelne Rundzellen sieht man ausserdem da und dort im gewucherten Intimagewebe rerstreut. Peripher folgt dann die als gewellte Linie hervortretende Elastica und auf diese die normalbreite Muscularis.

Die Adventitia zeigt mehrere zum Theil mit einander confluirende knötchenartige Neubildungen, deren periphere Theile aus dichten Ansammlnngen von epitheloiden und Rundzellen, deren centrale Theile aus blassen hrümligen, faserigen und scholligen Massen und dunklen Zellkerntrümmern bestehen. Jedes einzelne Neoplasma ist von einer Hülle faserigen Bindegewebes umgeben, ringsum findet sich starke zellige Infiltration und Hyperämie. In dem kleinen noch erhaltenen Gefässlumen wie in den neugebildeten Blutränmen finden sich wohlerhaltene rothe und weisse Blutkörperchen (Fig.2).

2. Ein Schrägschnitt durch beide Vertebrales, an deren Vereinigungsstelle, zeigt das Lumen der rechten stark verengt, fast obliterirt durch starke Intimawucherang; dieselbe erscheint lateral am stärksten, medial am geringsten. Das neugebildete Gewebe ist anch hier theilweise hyalin degenerirt.

Die hyaline Partie umgiebt das Lumen als breiter Ring, dem lateral die spindelförmigen, auch vermindert färbbaren Zellen, von zahlreichen (dunklen) Rundzellen durchsetzt, sich anschliessen, medial folgt auf die hyaline Zore ein mondsichelförmiges Gebiet von unregelmässig ansgebuchteten Bluträumen, die von zahlreichen Rundzellen umgeben sind. Auf die Elastica folgt peripher medial eine breite Schicht von Muskelzellen, an die sich die Elastica des Nachbargefässes anschliesst. Lateral finden sich in die Media und Adventitia 
der rechten Vertebralis eingelagert, mehrere knötehenförmige Herde, von ähnlicher Beschaffenheit wie vorher beschrieben. Die Nelrose ist hier an verschiedenen Knötchen verschieden weit fortgeschritten. Ein kleines Vas yasorum zeigt ebenfalls eine beträchtliche Verengung seines Lumens und in mehreren concentrischen Ringen angeordnete Rundzelleninfiltration des angrenzenden Bindegewebes. Zwischen ihm und dem Hauptgefäss befindet sich ein nekrotisches Gebiet, an dessen Rande vereinzelte Riesenzellen liegen.

Die linke Vertebralis zeigt normalen Bau, nur ihre Adrentitia ist in der Nähe der rechten Vertebralis von einzelnen Rundzellen durchsetzt.

3. Ein Querschnitt durch den unteren Theil der Basilaris ergiebt ebenfalls beträchtliche Intimawucherung, doch ist das Lumen hier nicht so erheblich verengt, wie in der Vertebralis, die spindelförmigen Zellen sind spärlicher vor Rundzellen durchsetzt. Adventitia und zum Theil anch die Media sind durch stark nekrotisirte Neubildung zerstört. (Fig. 3).

4. Die Basilaris in ihrem mittleren Theile hat ein bedeutend weiteres Lumen als in dem vorher beschriebenen Präparate. Die Intima ist hier nur einseitig verdiclit.

Entsprechend der Stelle breitester Intimawncherung findet sich das Gebiet der Media und Adventitia einnehmend ein schmaler, sichelförmiger, zum grössten Theil aus Kerndetritus bestehender Herd, ein der Nekrose verfallenes Neoplasma.

Elastinfärbung nach Weigert an entsprechenden Präparaten lässt eine vielfache Zerfaserung der Membrana fenestrata erkennen.

Figur 4 veranschaulicht einen Theil der Gefässwand in stärkerer Vergrösserung. Die Elastica besteht hier aus einer breiteren peripheren Lamelle, und $7-8$ central daran sich anschliessenden feineren Lamellen: sie alle haben den charakteristisch welligen Verlauf.

Hervorzuheben ist ferner die Endarteriitis der Arteria spinalis anterior, die fast zur vollständigen Obliteration des Gefässes geführt hat. (Fig. 5). Media und Adventitia sind kleinzellig infiltrirt.

Bei Durchmusterung der Brücke und des verlängerten Markes fanden sich nun zwei Erweichungsherde, beide rechts gelegen; der obere hat seine grösste Ausdehnung etwa der Obersteiner'schen Ebene k entsprechend, reicht cerebralwärts bis zwischen Ebene $m$ und $n$, caudalwärts nicht ganz bis zur Ebene i. (Zu vergleichen Fig. 6).

Entsprechend der grössten Ausdehnung des Herdes erscheint in Präparaten, die mit der Markscheidenfärbung nach Wolters gefärbt sind, das Gebiet der rechten Pyramide und angrenzenden Faserbündel der Brücke fast völlig ungefärbt und in ein lockeres Maschenwerk umgewandelt. Im Centrum des Herdes ist das brüchige Gewebe theilweise beim Schneiden ausgefallen.

An der Peripherie sieht man die erhaltenen Axencylinder (an Urancarminpräparaten) stark gequollen. Bei der Reaction nach Marchi sieht man die charakteristischen schwarzen Schollen und Fettkörnchenzellen im Erwe ichungsgebiete.

Der zweite Erweichungsherd betrifft das Pyramidengebiet der rechten 
Seite zwischen den Ebenen d und e. (cf. Fig. 7). Die betreffende Partie erscheint hier wie siebartig durchlöchert, die Axencylinder sind hier theils geschwunden, theils stark gequollen. Das Neurogliagewebe ist noch gut erhalten. Die Lichtung der Nervenfasern erstreckt sich in dorsaler Richtung bis über das untere Drittel der Schleife. Die vom Schnitt getroffenen perivasculären Räume sind theilweise auch ausserhalb des Erweichungsherdes erweitert.

Ferner wurde constatirt eine Perineuritis des Chiasma opticum, die Nervenfasern nur in mässiger Zahl geschwunden.

Die Pia und Arachnoidea an Pons und Medulla oblongata sind allenthalben mehr oder minder stark kleinzellig infiltrirt.

Die Untersuchung der Hirnrinde ans Stirnwindung, Centralwindungen. und Schläfenwindung ergab durchaus den Befund, wie man ihn bei allgemeiner progressiver Paralyse zu erbeben pflegt. Hervorgehoben sei, dass am Stirnhirn die Tangentialfasern völlig geschwunden, das superradiäre Flechtwerk bis auf vereinzelte Fasern geschwunden ist. Völlig geschwunden sind die Tangentialfasern auch im Schläfenlappen und in den Centralwindungen. Die Gefässchen der Hirnrinde sind theilweise hyalin entartet, die perivasculäre kleinzellige Infiltration ist nur ganz gering im Vergleich zu derjenigen, wie wir sie in der Medulla oblongata, Brücke und Hirnschenkeln fanden.

Wir lassen jetzt die Krankengeschichte unseres zweiten Falles folgen:

Friedrich Otto B., Schachtmeister, 24. December 1863 geboren, verheirathet, Vater von 6 Kindern, an 2. Juli 1900 in Sonnenstein aufgenommen. Seit Weihnachten 1899 matt, arbeitsunlustig. Schlief viel am Tage. Redete einmal viel vor sich bin, sah "schwarze Männer", hörte, dass seine Frau seine Mutter "Hure" nannte. Einmal Sohwindelanfall, danach sagt er, schwarze Männer hätten ihm auf dem Gesicht herumgetanzt. In letzter Zeit vor der Aufnahme wortkarg, unordentlich in seiner Kleidung, liess Urin ins Zimmer, ins Bett, liess Koth in die Hosen, ass viel, liess sich Zechprellereien zu Schulden kommen, nahm im Restaurant Chololade und Cigarren aus den Kästen, zeigte zuweilen kindisches Benehmen. 1898 wurde er wegen „einer Krankheit am Glied" operirt, in letzter Zeit Nachlass der Potenz. Einmal im Jahre 1898 soll es ihm den Mund auf die Seite gezogen haben, sodass er nicht sprechen konnte; der Anfall ging rasch vorüber.

\section{Status vom 4. Juli 1900.}

Oertlich desorientirt, behauptet bis zum 6. Lebensjahre die Schule besucht zu haben in Schönhaide, im 7. Jahre wäre er nach Glauchau gegangen. Dort sei er gewesen bis Ende 1899, hätte Sehreiben, Lesen, Singen bis Ende vorigen Jahres gelernt. Seit 1889 sei er verheirathet.

Rechnet $13+17=24,10-7=2,100: 25=25$.

Ist meist indolent, manchmal widerstrebend, oft mehr, oft weniger benommen.

Keine Grössenideen.

Aus dem körperlichen Befund ist hervorzuheben: 
Am Wirbel wenig Haare. Pupillen different, rechte weiter als linke, beide lichtstarr, accommodative Reaction erhalten. Linke Nasolabialfalte in der Ruhe ein wenig verstrichen. Zunge eine Spur nach rechts abweichend.

Artikulatorische Sprachstörung.

Rachenreflex herabgesetzt.

Am Penis Narbe im dorsalen Theil der Uebergangsfalte, circumcidirtes Stück des Präputiums fehlt.

Grobschlägiges Zittern der Finger.

Tastsinn normal, Schmerzsinn stark herabgesetzt.

Rechter Patellarsehuenreflex vorhanden, linker fehlt fast vollständig.

Kein Romberg, Gang o. B.

Zunächst ruhig dement. Allmählig weiterer Rückgang der geistigen Fähigkeiten, euphorische Stimmung.

7. Februar 1901. Ziemlich acut ist ein enormer Erregungszustand ausgebrochen mit stärkstem motorischen Drange, fabelhaften Grössenideen, Verworrenheit.

10. Februar. Zieht sich gefährliche Wunden zu, beschmutzt sie, reisst die Verbände herunter, muss beschränkt werden. Erhält dann zweitägig lange Dauerbäder, flüssige Nahrung.

13. März. Nachdem vor 8 Tagen das Camisol entfernt wurde, sucht sich Patient heate so rücksichtslos die Haut der Fersen in langen Lamellen herunter zu ziehen, dass eine Blutung daselbst eintrat. Camisol. Patient andauernd stark erregt, arbeitet in sinnloser und rücksichtsloser Weise mit Armen und Beinen unher, schreit verworreno Reden, ist unrein, nährt sich mangelhaft, verfällt körperlich und geistig von Tag zu Tag mehr und mehr.

Zieht sich ein Haematoma traumaticum der Stirn zu. (2. Mai).

13. Mai 1901. Exitus letalis unter Zeichen von Herzschwäche.

Aus dem Sectionsbericht sei hervorgehoben:

Subperiostales Haematom der Stirn, braune Atrophie des Herzens. Aorta im aufsteigenden Theile mit kleinen atheromatösen Einlagerungen, sonst glatt.

(Prostata und Samenbläschen o. B.). Hoden taubeneigross, zeigen keine geschrumpften Partieen.

Penis zeigt am inneren Blatt der oedematös geschwollenen Vorhaut eine linsengrosse, warzenförmige Excrescenz.

Kopfböhle: Schädeldach dünn, wenig Diploë, an manchen Stellen gar keine.

Keine Verwachsungen zwischen Schädeldach und Dura.

Innenseite der Dura zeigt über dem linken Stirnlappen und rechten Sohläfenlappen feine flächenhafte Blutangen.

Kein Hydrocephalus externus. Weiche Hirnhäute etwas trüb, an ganz vereinzelten Stellen mit der Hirnrinde verwachsen.

Stirnhirnwindungen mässig atrophirt.

Ependym aller Ventrikel granulirt. Seiten- und 3. Ventrikel nicht unerheblich erweitert.

Direct neben dem rechten Tractus opticus und zwar lateralwärts ron 
demselben liegt offenbar in Verbindung mit der Carotis dextra ein kirschkerngrosser, gelber, mittelhart anzufühlender Knoten.

Hirnsubstanz mässig feucht, anämisch.

Auch nach der Section wurde die Diagnose auf: Progressive Paralyse gestellt.

Ein Frontalschnitt durch den im Sectionsprotocoll erwähnten kirschkerngrossen Tumor und seine Umgebung liefert das folgende Bild (cf. Fig 8).

Lateral von dem querdurchschnittenen Chiasma opticum folgt zunächst jener Tumor, und diesem wieder die querdurehschnittene rechte arteria carotis interna. Der Tumor, durch die vorausgegangene Bebandlung etwas geschrumpft, erscheint auf seinem grössten Durchschnitt eliptisch. Das zellenarme, im Centrum gelegene nekrotische Gewebe lässt eine theils netzförmige, theils homogene Struktur erkennen. Die Richtung der Fasernordnung und ibre Dichtigkeit ist an verschiedenen Stellen verschieden, sodass sich unschwer erkennen lässt, dass der Tumor sich aus mehreren confluirten Knötchen zusammensetzt. Hier und da sind noch ganz blass gefärbte runde und ovale bläschenförmige Kerne zu erkennen.

Umgeben jst dieser eben beschriebene centrale nekrotische Theil von einem Ringe dunkel gefärbter Rundzellen; bis eine Strecke in den kernarmen (käsigen) Theil hinein schieben sich einzelne zapfenförmige Herde von Rundzellen vor. Stellenweise finden sich auch in der kernreichen Zone nekrotische Partieen.

Der Tumor wird von einer Zone derben, faserigen Bindegewebes eingeschlossen, die auf van Gieson'schen Präparaten besonders deutiich hervortritt.

Die Tunica intima der benachbarten arteria carotis interna ist an ihrer medialen dem Tumor zugekehrten Seite beträchtlich verdickt und gewuchert; an der gegenüberliegenden Seite ist die Intima von gewöhnlicher Stärke. Die Neubildung entspricht völlig der Heubner'schen Endarteriitis deformans. Die Elastica umgiebt als einfache geschlängelte Linie den Intimaring. Die Muscularis ist allenthalben wohl erhalten, von gewöhnlicher Stärke; die Adventitia nur in der Umgebung der Capillaren mit Rundzellen reichlicher durchsetzt (cf. Fig. 9).

An den vom Schnitt mitgetroffenen Theilen des Linsenkerns und Thalamus opticus fällt eine hochgradige perivasculäre kleinzellige lnfiltration auf, die an Intensität immer mehr abnimmt, je mehr man- sich von der Basis cerebri in dorsaler Richtung entfernt.

Auch auf das Perineurium des Chiasma erstreckt sich die entzündliche Infiltration.

Fassen wir die Ergebnisse unserer Untersuchung zusammen, so handelt es sich in unserem 1. Falle um eine Erkrankung der basalen Hirnarterien, und zwar beschränkt auf einen Theil der Arteria vertebralis, der Basilaris und der beiden Cerebrales anteriores, eine Er- 
krankung, die nach Ansicht aller Autoren als besonders charakteristisch für Lues angesehen werden muss: die Arteriitis gummosa.

Sie ist hier verbunden mit Endarteritis, wie sie Heubner in seiner bekaunten Arbeit beschrieb; diese Endarteritis fand sich in unserem Falle auch an kleineren Gefässstämmchen innerhalb der Meningen und besonders stark fortgeschritten an der Arteria spinalis anterior, wo sie fast bis zur vollständigen Obliteration geführt hatte. Die Erweichungsberde im Pons und in der Hedulla oblongata resultiren ans der Behinderung der Bluteirculation in der Arteria basilaris und spinalis anterior.

Da das Gebiet der Carotis interna und ihrer Aeste völlig intact befumden worden sind, die Pia convexitatis fast völlig frei von entzündJichen Veränderungen war und auch die Gefässe der Hirnrinde selbst nur an einzelnen Stellen mässige perivasculäre kleinzellige Infiltrate, wie sie bei jeder gewöhnlichen Paralyse angetroffen werden, aufwiesen, so müssen die diffusen degenerativen Veränderungen der nervösen Elemente der Hirnrinde als rein primäre, typisch paralytische aufgefasst werden.

Der im zweiten Falle bei der Obduction gefundene Tumor zwischen Chiasma opticum und Arteria carotis interna ist ebenfalls zweifellos als spezifisch anzusehen: er ist nichts anderes als ein typisches Gumma.

Die hier vorliegenden Gefässveränderungen beschränkten sich auf die allernächste Umgebung der Neubildung; es fand sich eine Endarteriitis deformans der Carotis interna dextra, die bei der geringfügigen Verengung des Lumens nur zu einer unerheblichen Circulationsstörung geführt haben konnte.

Beide Male fanden sich diese spezifischen Veränderungen als Nebenbefunde bei Sektionen von Fällen, die klinisch vollständig das Bild der allgemeinen progressiven Paralyse geboten hatten. Es handelte sich beide Male um eine gleichmässig fortschreitende Verblödung; in beiden Fällen entsprach die Krankheitsdauer ungefähr der Durchschnittsdauer der typischen Paralyse: im ersten Falle betrug sie nur 2, im anderen $11 / 2$ Jahre.

Dass in beiden Fällen die spezifischen Veränderungen der Gehirnbasis klinisch nicht mehr in den Vordergrund traten, ist bei den weit vorgeschrittenen allgemeinen Lähmungserscheinungen nicht weiter verwunderlich. Selbst die Degenerationsherde des ersten Falles in Brücke und Nedulla oblongata im Bereiche der Pyramiden konnten ja höehstens in Form von Paresen sich manifestiren, da in den regressiv 
veränderten Partien allenthalben noch eine grosse Anzahl von Nervenfasern und insbesondere Axencylindern erbalten geblieben waren.

Ein Augenspiegelbefund war in beiden Fällen nicht erhoben worden. Dass es sich in unseren Fällen um luetische Erkrankungen handelt, dafür spricht im ersten Falle schon der typische Sitz der Neoplasmen in und an den grossen Gefässen der Basis (besonders vertebralis und basilaris) und im zweiten Falle die Localisation des Tumors in der Umgebung des Chiasma und der Carotis verbunden mit einer specifischen Endarteriitis deformans circumscripta und basalen Meningitis.

Aehnliche Fälle von Combination der Hirnsyphilis mit progressiver Paralyse sind früher veröffentlicht von Rumpf, Westphal, Zambaco, Meyer, Haenel, Jolly, Wickel und neuerdings besonders von Binswanger, v. Rad, Nonne u. A.

In einem von Henneberg geschilderten Falle fand sich bei der Section als alleiniger spezifischer Hirnbefund dicht am Zusammenfluss der beiden Arteriae vertebrales eine erbsengrosse, gelbliche, geschwulstartige Verdickung der Wand der :Arteria basilaris, der vor dieser Stelle gelegene Theil derselben war aufgetrieben und enthielt einen ziemlich resistenten schwarzbraunen Thrombus, der sich eine kurze Strecke weit in beide Arteriae profundae cerebri fortsetzte. Alle übrigen Arterien normal, keine meningitischen Veränderungen, keine Erweichungsherde der Brücke.

Ich citire diesen Fall nur wegen der unserem ersten Falle ähnelnden Localisation der gummösen Arteriitis, progressive Paralyse kam hierbei nicht in Frage.

Binswanger spricht sich in seinen Beiträgen zur Pathogenese und differentiellen Diagnose der progressiven Paralyse dabin aus: "Man kann, wie besonders in dem Westphal'schen Falle frische gummöse Prozesse und arteriitische Veränderungen im Gehirn und seinen Hänten neben den diffusen degenerativ atrophischen Hirnrindenveränderungen der Paralyse auffinden und wird dann zu dem Schlusse gelangen, dass das syphilitische Virus gleichzeitig eine doppelte Einwirkung ausgeübt hat.

Es regt dieselbe die Entwickelung infectiöser Granulationsgeschwülste an und wirkt (durch die Stoffwechselprodukte des hypothetischen Syphilisbacillus?) chemisch schädigend auf die functionstragende Nervensubstanz. In den beiden von mir mitgeteilten Fällen ist diese doppelte pathogenetisch und anatemisch durchaus verschiedenartige Wirkungsweise leichter erkennbar, indem die spezifisch gummöse Erkrankung von der später nachfolgenden Paralyse durch eiuen langen Zeitraum getreunt war." 
In dem ersten Binswanger'schen Falle handelte es sich um ein. spindelförmiges Syphilom in dem einen Oculomotorius, in dem zweiten Falle um zwei alte derbe von Spindei- und Rundzellen durchsetzte knotige Verdickungen in der Falx cerebri and in der Arteriia basilaris, beide Male combinirt mit progressiver Paralyse.

Neuerdings sagt Erb in seinen „Bemerkungen zur pathologischer Anatomie der Syphilis des centralen Nervensystems" von den sogen. primären parenchymatösen Degenerationen an Nervenfasern und Ganglienzellen, mit oder ohne gleichzeitige Gliawucherung, chronischen Myelitis, fleckweisen Sklerosen, Strangdegenerationen and Kernatrophien:

„Es ist zu untersuchen, ob solche Dinge sich neben den sicher syphilitischen Veränderungen (in unmittelbarer Nähe derselben, oder auch entfernt davon im Nervensystem) also in Combination mit denselben finden; ob sie vorwiegend oder doch recht häufig bei Leutem vorkommen, die früher syphilitisch waren, resp. ob bei den von ihnen betroffenen Individuen sich mit besonderer Häufigkeit Syphilis in der Vorgeschichte nachweisen lässt, vorausgesetzt, dass andere ursächliche Momente fehlen. Wenn sich die anerkannt spezifischen ¿Läsionen neben solchen nicht spezifischen finden, wenn dabei stets, oder doch sehr häufig Syphilis vorausgegangen und eine andere Infectionskrankbeit oder eine Intoxication nicht nachweisbar ist, wäre es doch ein ganz unerlaubtes Maass von Skepsis, es wäre geradezu unverständig, die letzteren als nicht von der Syphilis ausgelöst zu betrachten."

Erb hat nun eine grössere Anzahl von Tabesfällen, die mit sicher syphilitischen Veränderungen combinirt waren, gesammelt und damit für die Tabes den Nachweis geliefert, oder wie er sagt, wenigstens "angebahnt, dass anscheinend einfache, "nicht spezifische" primäre Degenerationsvorgänge an den nervösen Elementen und Leitungsbahnen von der Syphilis ausgelöst werden."

Denselben Weg der Beweisführung, den Erb für die Tabes und ihre Beziehung zur Syphilis eingeschlagen hat, wird man auch für die progressive Paralyse einschlagen können und zwar wenigstens für die Fälle, wo sich unzweifelhaft spezifische gummöse Veränderungen combinirt mit den gewöhnlichen Befunden: der alJgemeinen Paralyse (Atrophien, Sklerosen der Ganglienzellen, Nervenfaserschwund u. s. w.) finden, Fälle, die Jolly mit dem Namen Pseudoparalysis syphilitica belegt und zu deren Casuistik meine zwei eben vorgetragenen Fälle einen weiteren kleinen Beitrag liefern sollten.

Meinem hochverdienten Chef, Herrn Geh. Medizinalrat Dr. Weber, erlaube ich mir auch an dieser Stelle für die Ueberlassung der Arbeit meinen ehrerbietigsten Dank auszusprechen. 


\section{Literatur.}

Oppenheim, Die syphilitischen Erkrankungen des Gehirns.

Erb, Bemerkungen zur pathologischen Anatomie der Syphilis des centralen Nervensystems. Deutsche Zeitschrift für Nervenheilkunde. 22. Bd.

Volland, Apoplestischer Insult in Folge eines Erweichungsherdes in der Brücke und spätere Dementia paralytica. Arch. f. Psych. u. Nervenkrankh. 32. Bd. 3. Heft.

B a umgarten, Ein Fall von verbreiteter obliterirender Entzündung der Gehirnarterien mit Arteriitis und Periarteriitis nodosa gummosa cerebralis nebst Bemerkungen über Hirnarteriosclerose und die als Periarteriitis nodosa (Kussmaul nnd Maier) oder multiple Aneurysmen mittlerer und kleinerer Arterien (Meyer) bezeichnete Erkrankung. Virchow's Archiv. Bd. 76. Heft 1.

Dr. Th. Treitel und Prof. Dr. P. Baumgarten, Ein Fall von einseitiger temporaler Hemianopsie in Folge von syphilitischer (gummöser) Arteriitis cerebralis. Virchow's Archiv. Bd. 111, 11. Folge. Bd. 1, Heft 1.

Baumgarten, Ueber gummöse Syphilis des Gehirns und Rückenmarks, namentlich der Gehirngefässe und über das Verhältniss dieser Erkrankungen zu den entsprechenden tuberkulösen Affectionen. Virchow's Arch. Bd. 86. 8. Folge. Bd. 6, Heft 2.

Baumgarten, Ueber chronische Arteritis und Endarteriitis mit besonderer Berücksichtigung der sogenaunten "luetischen" Erkrankung der Gehirnarterien, nebst Beschreibung eines Beispiels von specifisch „syphilitischer (gummöser)" Entzündung der grossen Cerebralgefässe.

Non ne, Syphilis und Nervensystem.

Binswanger, Referat über "Nonne: Syphilis und Nervensystem". Monatsschrift für Psychiatrie und Neurologie. Bd. XI. 1902. S. $473 \mathrm{ff}$.

Binswanger, Die Abgrenzung der allgemeinen progressiven Paralyse. Berl. klin. Wochenschr. 1894, No. 49,50 u. 52.

Binswanger, Beiträge zur Pathogenese und differentiellen Diagnose der progressiven Paralyse. Virchow's Archiv. Bd. 154, Folge XV. Heft 4.

Siemerling, Zur Syphilis des Centralnervensystems. Arch. für Psych. und Nervenkrankh. XXII. Bd. Heft 1.

Rumpf, Die syphilitisohen Erkrankungen des Nervensystems.

Köppen, Beiträge zum Studium der Hirnrindenerkrankungen. Arch. für Psych. nnd Nervenkrankh. XXVIII. Bd. .3. Heft.

Haenel, Beitrag zur Kenntniss der Syphilis des Centralnervensystems. Arch. für Psych. und Nervenkrankh. XXXIII. Bd. Heft 2.

Jolly, Syphilis und Geisteskrankheit. Berl. klin. Wochenschr. 1901. No. 1. C. Wickel, Casuistische Beiträge zur Differentialdiagnose zwischen Lues cerebri diffusa und Dementia paralytica nebst einem anatomischen Befunde. Arch. f. Psych. Bd. XXX. Heft 2. 
194 Dr. Rentsch, Ueber zwei Fälle v. Dementia paralytica mit Hirnsyphilis.

Angelo Cippolina, Contributo allo studio dell' endoarterite sifilitica. Neurol. Centralblatt 1903. No. 11.

Wendeler, Zur Histologie der syphilitischen Erkrankungen der Hirnarterien.

Deutsch. Arch. f. klin. Medic. Bd. LV.

Henneberg, Fall von Thrombose der Arter. bas. Neurol. Centralblatt.

XIX. Jahrgang. No. 23.

Birch-Hirschfeld, Specielle pathologische Anatomie.

von Rad, Ueber einen Fall von juveniler Paralyse. Archiv für Psych. und Nervenkrankh. Bd. 30. Heft 1.

\section{Erklärung der Abbildungen (Taf. IV).}

Figur 1. Arteriitis gummosa der rechten Arteria vertebralis und der Arteria basilaris.

Figur 2. Arteria vertebralis dextra unmittelbar vor dem Uebergang in die Arteria basilaris quer durchschnitten. Peri- et mesarteriitis gummasa. Starke Intimawucherung, die fast bis zur Obliteration des Lumens geführt hat. Die Intima ist stark vascularisirt.

Figur 3. Arteria basilaris, mittlerer Theil. Peri- et mesarteriitis gummosa, endarteriitis.

Figur 4. Arteria basilaris. Elastinfärbung nach Weigert.

Figur 5. Arteria spinalis anterior durch Intimawucherung fast obliterirt. Rundzelleninfilteration der Adventitia. Meningitis spinalis.

Figur 6. Degenerationsherd im Pons.

Figur 7. Degenerationsherd in der Medulla oblongata. Beide Herde in Figur 6 und 7 sind rechtsseitig.

Figur 8. Frontalschnitt dureh das Chiasma opticum und die Arteria carotis interna. Zwischen beiden liegt das in seinem grössten Durchmesser durchschnittene Gumma. Die Intima der Carotis interna ist einseitig verdickt, und zwar auf der dem Gumma benachbarten Seite.

Fg ur 9. Carotis interna. Endarteriitis eircumseripta. 